\title{
Awareness of FMF among Caregivers
}

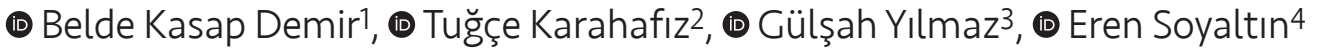 \\ 1'izmir Katip Çelebi University Faculty of Medicine, Department of Pediatric Nephrology and Rheumatology, İzmir, Turkey \\ 2Simav District Health Directorate, Kütahya, Turkey \\ ${ }^{3}$ Akseki Community Health Center, Antalya, Turkey \\ 4University of Health Sciences Turkey, Tepecik Training and Research Hospital, Clinic of Pediatric Nephrology, İzmir, Turkey
}

\begin{abstract}
Aim: Familial Mediterranean Fever (FMF) is one of the most frequent genetic diseases with a frequency of 1/1,000 in our country. We aimed to assess awareness of FMF among caregivers of patients with and without FMF.

Materials and Methods: Caregivers of FMF patients (group 1) and other caregivers who were in hospital for other reasons (group 2) were given a questionnaire regarding FMF and the results were compared between the 2 groups.

Results: The mean ages were similar between group $1(n=142)$ and group $2(n=207)$. Female gender and lower educational status were more frequent in group 1. Group 1 was more aware that recurrent fever, joint pain, abdominal pain, and its occurrence in attacks are seen in FMF; and also that the disease is inherited rather than contagious; that a specific treatment exists; that the risk of kidney and heart failure increases when not treated; that attacks are triggered by sleeplessness, tiredness or emotional stress; that colchicine does not provide complete recovery; and that diarrhea is the most common side effect of colchicine $(p<0.05)$. However, awareness about chest pain as a feature, infertility as a complication, non-adherence as a trigger for the disease and the statement that "patients feel healthy between attacks" were similar ( $p>0.05$ ). Interestingly, group 2 was more aware that different treatment options exist $(p=0.04)$. The total score was higher in group 1 and in participants who only graduated from secondary school or less $(p<0.01)$.
\end{abstract}

Conclusion: Being a caregiver of a patient was associated with a higher level of awareness of FMF regardless of education level, however, they still need to be informed about current developments.

Keywords: Familial Mediterranean Fever, awareness, caregivers

\section{Introduction}

Familial Mediterranean Fever (FMF) is an autosomal recessive disease characterized by recurrent episodes of fever, peritonitis, pleuritis, arthritis and erysipelas-like skin lesions. It is the most common form of hereditary autoinflammatory disorders (1,2). The estimated prevalence of FMF is $1 / 1,000$ and the carrier rate is $1: 5$ in our country, Turkey $(1,3)$.

The most common complication of FMF is secondary amyloidosis. To avoid complications, both clinicians and the general population should be well-informed. With the increased awareness of physicians, the diagnosis of the disease and the prescription of colchicine, which has been reported as the standard of care for prevention of attacks and secondary amyloidosis, has increased. As a result, the rates of patients with amyloidosis have decreased over time (4). In a previous study, it was indicated that only $18 \%$ of the population in the third largest city of our country where this study was conducted were aware of this disease (5).

In this study, we aimed to evaluate and compare the awareness levels of the caregivers of those patients diagnosed 
with FMF and the caregivers of other children admitted to outpatient clinics. This study was carried out via a face-toface questionnaire conducted by volunteer medical school students. In this way, it was planned to also raise awareness and more thoroughly inform caregivers about FMF by giving the correct answers to the questionnaire questions at the end of the interview to all study participants.

\section{Materials and Methods}

This study was approved by the Izmir Katip Çelebi University, Non-Invasive Clinical Research Ethics Committee (17.01.2018/16). The caregivers of those patients who were being followed up in pediatric nephrology and pediatric rheumatology outpatient clinics with a diagnosis of FMF and the caregivers of other outpatients between April 2018 and October 2018 were enrolled in this study. All participants completed a questionnaire including personal information about age, gender, occupational and educational status and 17 additional questions about complaints regarding FMF disease, its way of transition, its treatment options and possible complications. At the end of the questionnaire, they all agreed to the statement that their data could be used in scientific studies and signed the form. Educational status was categorized as "graduated from elementary school", "graduated from secondary school", "graduated from high school", or "graduated from academy/university". While selecting participants other than the caregivers of FMF patients, we asked the question "Have you ever heard about FMF disease?" and continued with the questionnaire with those who answered the question positively. The questionnaire was conducted by volunteer medical school students via a face-to-face method. The students were informed about the answers of the questionnaire and they were asked to subsequently share the correct answers with the participants.

The first question was about any first-degree relatives of the participants diagnosed with FMF including their own selves. All except one of the following questions had the two options of "yes" or "no". The next five questions asked whether the patients had recurrent fever, abdominal pain, joint pain, chest pain or complaints that appear with attacks. The following two questions were about the transition of the disease. They asked whether FMF was a contagious disease or whether it was inherited and consanguinity played a role. The rest of the questions were about treatment options and complications. They asked whether there was a drug specifically used in FMF; whether FMF caused renal failure, cardiac failure or infertility; whether attacks were triggered by sleeplessness, tiredness, emotional stresses and/or by missing the treatment; whether patients felt healthy between the attacks; whether there was a complete cure with colchicine; and whether there was any other treatment option. The only question having two different options was the one which asked about the most common side-effect of colchicine being either vomiting or diarrhea. One point was given to each correct answer and a "total score" was calculated for each participant.

The caregivers of our patients with FMF constituted the study group (group 1) and the others formed the control group (group 2). However, among the control group, those individuals diagnosed with FMF themselves or those who had first-degree relatives diagnosed with FMF whom they take care of were transferred into the study group. While answering the questions, participants in group 1 were asked to evaluate the features of the disease in general rather than personally. The correct answers and total scores were compared between the groups.

\section{Statistical Analysis}

Statistical analyses were performed using SPSS 22.0 (SPSS Inc., Chicago, IL). Descriptive statistics are shown in mean \pm standard deviation and frequency format. Continuous variables between the groups were compared by Student's t-test, and categorical variables were compared with chi-square test. A " $p$ " value of $<0.05$ was considered significant in all statistical evaluations.

\section{Results}

A total of 349 volunteers participated in this study. Of these, 114 were the caregivers of patients with FMF and 235 were the caregivers of other outpatients. Twenty-eight of the latter group (12\%) had a relative with FMF whom the individual took care of or they had been diagnosed with FMF themselves. Thus, 28 individuals were transferred to group 1. Finally, group 1 consisted of 142 and group 2 consisted of 207 patients.

When the demographic findings were compared, the ages were similar between the groups. Female gender and an education level of only graduating from secondary school or less was more common in group 1 (Table I). When the rates of correct answers were compared between the groups, the

Table I. Comparison of the demographic findings between the groups

\begin{tabular}{|l|l|l|l|}
\hline & $\begin{array}{l}\text { Group 1 } \\
(\mathbf{n = 1 4 2 )}\end{array}$ & $\begin{array}{l}\text { Group 2 } \\
\mathbf{( n = 2 0 7 )}\end{array}$ & p-value \\
\hline Age (Year) & $37.68 \pm 8.11$ & $36.46 \pm 10.2$ & 0.238 \\
\hline Gender (Female) & $103(73 \%)$ & $123(59 \%)$ & $\mathbf{0 . 0 4}$ \\
\hline $\begin{array}{l}\text { Education (Secondary } \\
\text { education level or less) }\end{array}$ & $107(75 \%)$ & $41(20 \%)$ & $<\mathbf{0 . 0 1}$ \\
\hline
\end{tabular}


rate of awareness that recurrent fever, abdominal pain and joint pain are observed in FMF was higher in group 1, while expectancy for chest pain was similar between the groups. Participants in group 1 were more frequently aware that FMF occurs in attacks, is not a contagious disease being transmitted genetically, and that a specific drug exists for the treatment of FMF. In addition, group 1 was more frequently aware that FMF may cause renal and cardiac failure. However, the rate of awareness that FMF may cause infertility was similar between the groups. Group 1 was more likely to be aware that sleeplessness, tiredness and emotional stresses may trigger attacks. Participants in both groups were similarly aware in high rates that missing treatment may trigger attacks and that patients feel healthy between attacks. Group 1 was more aware that patients cannot be totally cured with colchicine and the most common side effect of colchicine is diarrhea, as expected. However, contrary to expectations, group 2 was more aware that there are different treatment options other than colchicine (Table II).

Group 1 had a significantly higher total score when compared with group 2. When educational status was analyzed, there were 148 patients who had only graduated from secondary school or less and there were 201 participants who had continued their education beyond secondary school. The "total score" was interestingly higher in those participants who had only graduated from secondary school or less $(12.87 \pm 2.74$ vs. $11.66 \pm 2.7 ; p<0.01)$. Explaining this situation, it was observed that those participants belonging to group 1 were more frequently from those who had only graduated from secondary school or less (72\% vs. 17\%, $p<0.01)$.

\section{Discussion}

This study, based on a questionnaire carried out by volunteer caregivers determined that individuals who were

\begin{tabular}{|c|c|c|c|c|}
\hline Questions & $\begin{array}{l}\text { Group } 1 \\
(n=142) \\
(n, \%)\end{array}$ & $\begin{array}{l}\text { Group } 2 \\
(n=207) \\
(n, \%)\end{array}$ & p-value & $\begin{array}{l}\text { Previous study } \\
(n=524) \\
(\%)\end{array}$ \\
\hline Recurrent fever & $135(96)$ & $161(78)$ & $<0.01$ & 84 \\
\hline Abdominal pain & $138(97)$ & $170(82)$ & $<0.01$ & 47 \\
\hline Joint pain & $137(97)$ & $141(68)$ & $<0.01$ & 54 \\
\hline Chest pain & $93(66)$ & $115(56)$ & 0.067 & 25 \\
\hline Appears with attacks & $139(98)$ & $152(74)$ & $<0.01$ & \\
\hline Contagious & $135(95)$ & $160(77)$ & $<0.01$ & \\
\hline Inherited and consanguinity play a role & $121(86)$ & $141(68)$ & $<0.01$ & 78 \\
\hline Causes renal failure & $134(94)$ & $121(58)$ & $<0.01$ & 78 \\
\hline Causes cardiac failure & $86(61)$ & $103(50)$ & 0.042 & 26 \\
\hline Causes infertility & $69(51)$ & $93(46)$ & 0.412 & $\begin{array}{l}\text { Male infertility: } 28 \\
\text { Female infertility: } 42\end{array}$ \\
\hline Specific medication exists for FMF & $133(99)$ & $160(77)$ & $<0.01$ & 72 \\
\hline $\begin{array}{l}\text { Sleeplessness, tiredness and stress trigger } \\
\text { attacks }\end{array}$ & $127(89)$ & $160(78)$ & 0.009 & $\begin{array}{l}\text { Sleeplessness: } 56 \\
\text { Tiredness: } 61 \\
\text { Emotional stress: } 69 \\
\text { Infections: } 63\end{array}$ \\
\hline Non-adherence to treatment triggers attacks & $129(91)$ & $182(89)$ & 0.522 & 81 \\
\hline Patients feel healthy between attacks & $91(64)$ & $127(62)$ & 0.727 & \\
\hline Total cure with colchicine? & $90(64)$ & $102(50)$ & 0.012 & \\
\hline Most common side effect? Vomiting? Diarrhea? & $99(74)$ & $106(53)$ & $<0.01$ & \\
\hline $\begin{array}{l}\text { Is there any different treatment option other } \\
\text { than colchicine? }\end{array}$ & $66(49)$ & $133(65)$ & 0.004 & \\
\hline Total score & $13.54 \pm 2.19$ & $11.24 \pm 2.76$ & $<0.01$ & \\
\hline
\end{tabular}


diagnosed with FMF or those who were taking care of a patient diagnosed with FMF were more aware of the disease, as expected. However, we observed that these caregivers were not well-informed that patients may have chest pain during attacks, FMF may cause infertility or that patients feel healthy between attacks. In addition, it was demonstrated that regardless of their level of education, responsibility for the primary health care of a patient with FMF provided a higher level of knowledge about the disease.

In our study, the mean ages of the participants in both groups were similar. However, female gender was more frequent and lower educational status was more common in group 1. A previous study was conducted on volunteers visiting a mega shop in our city (excluding those diagnosed with FMF and those who were medical doctors). It can be considered that this sample group included participants from all socio-economic levels. In this sense, it can be thought that the population of that study and our participants in group 2 are comparable.

FMF is characterized by recurrent episodes of fever and sterile inflammation resulting in abdominal pain caused by peritonitis, chest pain caused by pleuritis, and joint pain caused by arthritis. In the largest cohort from our country, the frequency of features of FMF were described as follows: fever $92.5 \%$, peritonitis $93.7 \%$, pleuritis $31 \%$, arthritis $47.4 \%$, arthralgia $49.7 \%$, erysipelas-like erythema $20.9 \%$, myalgia $39.6 \%$, protracted febrile myalgia $2.3 \%$, and protracted arthritis $2.6 \%$ (1). The most widely used adult criteria to diagnose FMF is the Tel Hashomer criteria (6). However, since the specificity was as low as $54.6 \%$ in children, the Yalcinkaya et al. (7) criteria was proposed for children in 2009. This set of criteria (7) and the most recent classification criteria (8) for FMF includes fever, abdominal pain, chest pain, arthritis and family history of FMF. Since the most frequent complaints reported in the nationwide cohort and proposed in the criteria were fever, abdominal pain, chest pain and arthralgia, we preferred to ask about these complaints in the questionnaire. Although myalgia has become a more recognizable feature of FMF (9), we did not put it among the options. As expected, group 1 was more aware that those patients with FMF have recurrent fever, abdominal pain and joint pain during attacks. However, information about chest pain was not well-known in group 1 with a rate of only $66 \%$, which was not different from the control group. This may be related to the fact that chest pain appears less frequently. In the previous study, although it is not possible to make a statistical analysis, the rate of awareness about the complaints of FMF were apparently lower, other than for recurrent fever, when compared to group 2 (Table II).
It is well-known that FMF is an autosomal recessive disease and consanguinity also plays an important role in its transition. In the largest nationwide survey from our country, the consanguinity rates ranged between 15\% and $26 \%$ in different regions (1). Group 1 was more aware about the transmission of the disease than group 2 , as well as the recent study participants.

Amyloidosis, which is the most undesirable complication of FMF, mainly affects the kidneys but it may also affect the heart (10). In addition, infertility is one of the most important long-term complications of FMF for both sexes. Infertility is more common in female patients, those with FMF disease onset $<20$ years, those with higher disease severity score, and colchicine non-response $(11,12)$. The caregivers of our patients were aware about the complications of FMF including renal and cardiac failure. However, we noticed that infertility was not well-known among them as it also was not well-known in the control group or among the participants of the previous study.

The most frequent triggering factors for FMF were defined as tiredness, emotional stresses, cold exposure and menstruation in females $(13,14)$. In addition, standing up for a long duration, long-duration travel or infection may precede attacks. Non-adherence to colchicine is another triggering factor for attacks (15). In our questionnaire, we asked whether sleeplessness, tiredness, emotional stresses and non-adherence to treatment may trigger the attacks. Group 1 was more aware of the triggering factors and all the participants were well-aware that non-adherence is another risk factor. This may be due in part to the way the question was asked. It is logical that non-adherence is an associated factor for risk.

In between episodes, affected individuals are usually symptom-free and feel healthy (16). However, only 64\% of the caregivers of our patients think in the same way. Interestingly, the rest of them thought that their children feel weak and tired most of the time even in non-attack periods.

FMF requires regular medication. Colchicine is an ancient drug proven to be effective in the prevention of recurrent inflammatory episodes and the development of amyloidosis in FMF (17). However, it has a narrow therapeutic margin. Its dose-dependent side-effects on the gastrointestinal system (increased motility, diarrhea, abdominal pain and vomiting) limit its use at toxic doses (17). Its most common adverse effect is diarrhea (18). As expected, almost all the participants in group 1 were aware that a specific treatment exists for FMF, and the other groups were able to give the correct answer in closed ranges. Group 1 answered the 
question correctly that colchicine cannot provide a complete cure and the most common side-effect is diarrhea.

In patients unresponsive to colchicine, interleukin-1 blockade provides an effective and safe way to suppress inflammation (17). It has become an increasingly popular treatment nowadays. Interestingly, not the primary caregivers of our patients with FMF, but the other participants were more frequently aware that there are other options apart from colchicine for FMF. We consider that we have not been able to inform the caregivers of our patients well enough. We had the opportunity to inform the caregivers and patients about the alternative options in colchicine resistant or intolerant cases.

\section{Study Limitations}

Our study has some limitations. The number and educational status of the patients in the groups were unequal. However, it was thought that our study can contribute to the literature, since the number of similar studies is limited. As a face-to-face method was used for the questionnaire, we had the opportunity to inform the families of the right answers at the end of the process. In addition, this study improved the ability of the students to design and perform a study and their own awareness about FMF. They also had the chance to observe the deficiencies of the primary caregivers in such a frequent disease.

\section{Conclusion}

Finally, we concluded that being close to a patient provides a higher level of awareness about FMF regardless of one's level of education. In addition, we noticed that even the primary caregivers can be unaware of well-known details and current developments. Thus, FMF caregivers and patients should be given accurate and up-to-date information whenever possible.

\section{Ethics}

Ethics Committee Approval: This study was approved by the İzmir Katip Çelebi University, Non-Invasive Clinical Research Ethics Committee (17.01.2018/16).

Informed Consent: Informed consent was obtained.

Peer-review: Externally peer-reviewed.

\section{Authorship Contributions}

Design: B.K.D., T.K., G.Y., E.S. Data Collection or Processing: T.K., G.Y., Analysis or Interpretation: B.K.D., E.S., Literature Search: B.K.D., Writing: B.K.D., T.K., G.Y., E.S.

Conflict of Interest: No conflict of interest was declared by the authors.
Financial Disclosure: The authors declared that this study received no financial support.

\section{References}

1. Tunca M, Akar S, Onen F, et al. Familial Mediterranean fever (FMF) in Turkey: results of a nationwide multicenter study. Medicine (Baltimore) 2005; 84:1-11.

2. Saatci U, Bakkaloglu A, Ozen S, Besbas N. Familial Mediterranean fever and amyloidosis in children. Acta Paediatr 1993; 82:705-6.

3. Yilmaz E, Ozen S, Balci B, et al. Mutation frequency of Familial Mediterranean Fever and evidence for a high carrier rate in the Turkish population. Eur J Hum Genet 2001; 9:553-5.

4. Akse-Onal V, Sag E, Ozen $S$, et al. Decrease in the rate of secondary amyloidosis in Turkish children with FMF: are we doing better? Eur J Pediatr 2010; 169:971-4.

5. Soylu A, Türkmen MA, Kavukçu S. The rate of awareness for Familial Mediterranean Fever for Urban Dwellers in Turkey. Turk | Rheumatol 2012; 27:275-77.

6. Livneh A, Langevitz $P$, Zemer $D$, et al. Criteria for the diagnosis of familial Mediterranean fever. Arthritis Rheum 1997; 40:1879-85.

7. Yalcinkaya F, Ozen S, Ozcakar ZB, et al. A new set of criteria for the diagnosis of familial Mediterranean fever in childhood. Rheumatology (Oxford) 2009; 48:395-8.

8. Gattorno $M$, Hofer $M$, Federici $S$, et al. Classification criteria for autoinflammatory recurrent fevers. Ann Rheum Dis 2019; 78:1025-32.

9. Demir K, Çarman KB, Kasap B. Concurrent protracted febrile myalgia syndrome in a child with diabetic ketoacidosis. Pediatr Diabetes 2012; 13:510-3.

10. Erken E, Erken E. Cardiac disease in familial Mediterranean fever. Rheumatol Int 2018; 38:51-8.

11. Atas N, Armagan B, Bodakci E, et al. Familial Mediterranean fever-associated infertility and underlying factors. Clin Rheumatol 2020; 39:255-61.

12. Yanmaz MN, Özcan AJ, Savan K. The impact of familial Mediterranean fever on reproductive system. Clin Rheumatol 2014; 33:1385-8.

13. Yenokyan G, Armenian HK. Triggers for attacks in familial Mediterranean fever: application of the case-crossover design. Am J Epidemiol 2012; 175:1054-61.

14. Karadag O, Tufan A, Yazisiz V, et al. The factors considered as trigger for the attacks in patients with Familial Mediterranean Fever. Rheumatol Int 2013; 33:893-7.

15. Ozen S, Demirkaya E, Erer B, et al. EULAR recommendations for the management of Familial Mediterranean Fever. Ann Rheum Dis 2016; 75:644-51.

16. Nir-Paz R, Ben-Chetrit E, Pikarsky E, Hassin D, Hasin Y, ChajekShaul T. Unusual presentation of familial Mediterranean fever: role of genetic diagnosis. Ann Rheum Dis 2000; 59:836-8.

17. Gül A. Treatment of familial Mediterranean fever: colchicine and beyond. Isr Med Assoc ) 2014; 16:281-4.

18. Verma S, Eikelboom JW, Nidorf SM, et al. Colchicine in cardiac disease: a systematic review and meta-analysis of randomized controlled trials. BMC Cardiovasc Disord 2015; 15:96. 